\title{
Fecal microbiota transplant via colonoscopy may be preferred due to intraprocedure findings
}

\author{
Janice M. Cho ${ }^{1 *}$, Laura Pestana ${ }^{1 *}$, Ryan Pardi ${ }^{2}$, Darrell S. Pardi ${ }^{2}$, Sahil Khanna $^{2}$ \\ ${ }^{I}$ Department of Internal Medicine and ${ }^{2}$ Division of Gastroenterology and Hepatology, Mayo Clinic, Rochester, MN, USA
}

Antibiotic treatment of Clostridium difficile infection (CDI) disrupts the normal gut flora and contributes to the risk of recurrent CDI, which is as high as $60 \%$ after 2 or more recurrences. ${ }^{1}$ Fecal microbiota transplant (FMT) yields high cure rates (87\%-90\%) with few serious adverse events and self-limiting adverse events. ${ }^{1-4}$ Higher success rates are seen with lower GI tract FMT delivery (91.4\%) than nasogastric or upper endoscopic delivery (82.3\%). ${ }^{5}$ At our institution, FMT is performed predominantly through colonoscopy. Donors are screened for depression, diarrhea, autoimmune disease, recent antibiotic exposure, colon polyps, pregnancy, detailed travel history, and GI tract history, among other considerations. If deemed appropriate for FMT, donors are subjected to blood and stool testing.

Endoscopic evaluation of the colon may be valuable for patients with recurrent CDI because of concomitant diagnoses such as IBD, which could predispose to recurrent CDI. In one study, 7 CDI patients (2.6\%) were found to have new-onset IBD diagnosed during FMT. ${ }^{5}$ The detection rate of other abnormalities during colonoscopy for FMT and the safety of interventions such as biopsies and polypectomy are not well investigated.

We explored the rate and nature of intraprocedure incidental findings. Herein, we outline the safety of biopsies and pol-

Received May 7, 2019. Revised May 9, 2019. Accepted May 10, 2019. Correspondence to Sahil Khanna, Division of Gastroenterology and Hepatology, Mayo Clinic, 200 First St SW, Rochester, MN 55905, USA. Tel: +1-507-266-4347, Fax: +1-507-284-0538, E-mail: Khanna.Sahil@mayo.edu

*These authors contributed equally to this study. ypectomy during colonoscopy for FMT.

This study was approved by the Mayo Clinic Institutional Review Board (IRB No. 14-005326) and patients were consented for follow-up. This retrospective cohort study evaluated patients who underwent FMT for recurrent CDI through colonoscopy between August 2012 and September 2015. Data were obtained from the electronic health record. Collected data included demographic characteristics, prior GI tract diagnoses, and colonoscopy findings, including macroscopic diagnoses and biopsy results.

Prior diagnoses were classified as IBD, microscopic colitis, colorectal cancer, colonic polyps, celiac disease, or other. Findings at FMT colonoscopy were classified as inflammation/ colitis, ulceration, stricture, polyp, normal, or other. Data on reasons for biopsy during colonoscopy were classified as abnormal macroscopic appearance, requested by referring provider, IBD surveillance, and suspected cytomegalovirus colitis, among others.

Biopsy results were classified as normal colonic mucosa, acute colitis, chronic colitis, microscopic colitis, IBD, or malignancy. For polyps, classifications were hyperplastic polyp, tubular adenoma with low-grade or high-grade dysplasia, tubulovillous/villous adenoma, sessile serrated adenoma, or malignant.

In August 2012, we established a program for FMT for management of patients with multiple recurrent and refractory CDI. Initially, the recipients identified a known stool donor. Due to logistics of finding a healthy known donor; a standard stool donor pool was established. These donors are known to 
the providers and undergo screening under the guidance of the provider. Donors who do not meet any exclusion criteria based on an initial review, are screened in the office for exclusion criteria with a detailed interview, and were then subjected to blood and stool testing. Patients are given the option to choose between a standard donor or identify a known donor (spouse, sibling, offspring, other household member, roommate or friend). Donor eligibility and screening criteria are similar for both standard and known stool donors. All donors undergo a detailed informed consent for donor screening and for stool donation. Eligible donors are instructed to report any interim symptoms and re-screened every 3 months. Screening and stool receipt logs are maintained. Standard donors are kept anonymous to recipients.

For stool preparation for fresh use, $50 \mathrm{~g}$ of donor stool is diluted in $250 \mathrm{~mL}$ of preservative free normal saline (used for intravenous injections). For stool preparation for frozen use, $50 \mathrm{~g}$ of donor stool is diluted in $250 \mathrm{~mL}$ of a solution (90\% preservative free normal saline used for intravenous injections and $10 \%$ glycerol used to preserve bacteria). The preparation to freeze is carried out in an anaerobic chamber and the solution is kept frozen at $-70^{\circ} \mathrm{C}$ until the day of the procedure and thawed at room temperature.

Patient with 3rd or greater episode of CDI, proven by a positive stool assay who have failed previous treatment with 1st line therapies for CDI (vancomycin, metronidazole, or fidaxomicin) were deemed eligible. Antibiotics used to treat CDI were stopped 24 hours prior to procedure. Patients underwent a standard bowel preparation and the procedure was carried out via a colonoscopy with $250 \mathrm{~mL}$ of diluted stool being instilled in the cecum.

The summary database was converted to a JMP file (SAS Institute Inc., Cary, NC, USA). Data analysis mainly involved descriptive statistics, and data were presented as frequency or percentage, or both, or as median and interquartile range where applicable. All authors had access to the study data and have reviewed and approved the final manuscript.

Overall, 234 patients (median [interquartile range] age, 56.6 [18.3-92.8] years) underwent FMT through colonoscopy for recurrent CDI (Table 1). Poor colonoscopy preparation was reported in $11.5 \%$ of patients. The majority of colonoscopies $(70.9 \%)$ were normal, while 36 patients $(15.4 \%)$ had inflammation or colitis on macroscopic examination (Table 1). Six patients $(2.6 \%)$ had ulceration and $2(0.9 \%)$ had strictures. Of patients with macroscopic findings of inflammation, 31 patients (86.1\%) either had a history of or eventually received a
Table 1. Patient Characteristics, Prior GI Tract Diagnoses, and Macroscopic Findings

\begin{tabular}{lc}
\hline Characteristic & Value $(\mathrm{n}=\mathbf{2 3 4})$ \\
\hline Age $(\mathrm{yr})$ & $56.6(18.3-92.8)$ \\
Race & $232(99.1)$ \\
White & $1(0.4)$ \\
African American & $1(0.4)$ \\
Hispanic & \\
Prior Gl tract diagnosis & $51(21.8)$ \\
IBD & $9(3.8)$ \\
Microscopic colitis & $1(0.4)$ \\
Colorectal cancer & $12(5.1)$ \\
Colonic polyps & $1(0.4)$ \\
Celiac disease & $2(0.9)$ \\
Other & $158(67.5)$ \\
None & \\
Macroscopic findings & $166(70.9)$ \\
Normal & $36(15.4)$ \\
Inflammation/colitis & $6(2.6)$ \\
Ulceration & $2(0.9)$ \\
Stricture & $19(8.1)$ \\
Colonic polyp & $5(2.1)$ \\
Other ${ }^{a}$ & \\
\hline
\end{tabular}

Values are presented as median (interquartile range) or number (\%).

${ }^{a}$ Other findings included pseudopolyps, fistulae, angioectasias, and reactive Peyer patches.

diagnosis of IBD.

Three patients received a new IBD diagnosis during FMT, and 7 had a new diagnosis of microscopic colitis. Five patients of those who had macroscopic inflammation on colonoscopy (13.9\%) had colitis secondary to CDI. Colonic polyps were found in 19 patients (8.1\%); 3 of these patients were younger than 50 years.

Overall, 158 patients (67.5\%) undergoing FMT had no known prior GI tract diagnoses (Table 1). During FMT colonoscopy, biopsies were taken for abnormal macroscopic appearance $(\mathrm{n}=19,8.1 \%)$, request by the ordering provider $(\mathrm{n}=41,17.5 \%)$, or for IBD surveillance $(n=6,2.6 \%)$ or to rule out cytomegalovirus colitis $(\mathrm{n}=4,1.7 \%)$.

Detailed endoscopic evaluation for polyp detection is typically not performed during FMT colonoscopy because of possible impairment of visualization from transplanted stool. Despite this caveat, 19 patients in our analysis had polyps, which were removed for 13 patients (68\%) at the time of FMT colo- 
noscopy. Of the 13 patients in whom one or more polyps were removed, 19 sub-centimeter polyps were seen which ranged from 2 to $6 \mathrm{~mm}$ in size and were removed with cold biopsy forceps (59.5\%) or a cold snare (31.5\%). One $17 \mathrm{~mm}$ polyp was seen which was removed with a saline lift infection and hot snare removal technique. These polyps were seen in the cecum, transverse colon, descending colon and the rectum. No complications happened during these polypectomies. Future removal was planned for the other 6 patients (32\%). Pathologic findings included hyperplastic polyps (46\%) and tubular adenoma with low-grade (38\%) or high-grade (8\%) dysplasia, as well as malignancy (8\%). Colon cancer was newly diagnosed in 2 patients. No adverse events were reported post-FMT, including for patients with biopsy or polypectomy, or both.

During colonoscopy to perform FMT, important intra-procedural findings were not uncommon. Furthermore, biopsies and polypectomies were performed safely with no adverse events during FMT.

A paucity of literature reports on incidental findings during FMT colonoscopy. The value in discovering these incidental pathologic disorders is considerable because their identification has led to new diagnoses (i.e., IBD and microscopic colitis) with earlier therapeutic intervention for these patients. In addition, these patients would likely have ongoing diarrhea, and if an alternative diagnosis was not made at colonoscopy, they would likely have been retested for CDI. A subset of patients would have tested positive due to a prolonged carrier state, leading to clinical mismanagement such as ongoing antibiotic treatment. Moreover, the identification of neoplastic polyps and early cancers allowed for removal before more advanced lesions could develop.

Biopsies collected during colonoscopy have not been associated with major complications. Minor complications, including self-limited bleeding and pain, have been reported in a small percentage of patients. ${ }^{7}$ Although there may be some concern that performing biopsies or polypectomies at the time of FMT could lead to an increased risk of infectious complications, no adverse effects from these interventions were observed in our study. Given this favorable risk profile, biopsies and polypectomy during FMT can be safely performed.

Our study has some limitations due to its observational nature. As with all retrospective studies, observations were limited to the detail of documentation in the health record.

In conclusion, incidental pathologic findings are not uncommon during FMT colonoscopy. FMT through colonoscopy may be preferable to other FMT administration routes because bi- opsy and polyp removal can be performed safely and efficiently. Important diagnoses such as IBD and colon cancer can be made during FMT colonoscopy, which can substantially alter clinical management.

\section{FINANCIAL SUPPORT}

The authors received no financial support for the research, authorship, and/or publication of this article.

\section{CONFLICT OF INTEREST}

No potential conflict of interest relevant to this article was reported.

\section{AUTHOR CONTRIBUTION}

Conceptualization: Pardi DS, Khanna S. Methodology: Cho JM, Pestana L, Pardi DS, Khanna S. Data acquisition: Cho JM, Pestana L, Pardi R. Formal analysis: Cho JM, Pestana L, Pardi R, Pardi DS, Khanna S. Writing - original draft: Cho JM, Pestana L. Writing - review and editing: Cho JM, Pestana L, Pardi R, Pardi DS, Khanna S. Approval of final manuscript: all authors.

\section{REFERENCES}

1. Kassam Z, Lee CH, Yuan Y, Hunt RH. Fecal microbiota transplantation for Clostridium difficile infection: systematic review and meta-analysis. Am J Gastroenterol 2013;108:500-508.

2. van Nood E, Vrieze A, Nieuwdorp M, et al. Duodenal infusion of donor feces for recurrent Clostridium difficile. N Engl J Med 2013;368:407-415.

3. Cammarota G, Ianiro G, Gasbarrini A. Fecal microbiota transplantation for the treatment of Clostridium difficile infection: a systematic review. J Clin Gastroenterol 2014;48:693-702.

4. Rossen NG, Fuentes S, van der Spek MJ, et al. Findings from a randomized controlled trial of fecal transplantation for patients with ulcerative colitis. Gastroenterology 2015;149:110118.

5. Khoruts A, Rank KM, Newman KM, et al. Inflammatory bowel disease affects the outcome of fecal microbiota transplantation for recurrent Clostridium difficile infection. Clin Gastroenterol Hepatol 2016;14:1433-1438.

6. Tariq R, Weatherly R, Kammer P, Pardi DS, Khanna S. Donor screening experience for fecal microbiota transplantation in patients with recurrent C. difficile infection. J Clin Gastroen- 
terol 2018;52:146-150.

7. Yao MD, von Rosenvinge EC, Groden C, Mannon PJ. Multiple endoscopic biopsies in research subjects: safety results from a National Institutes of Health series. Gastrointest Endosc 2009;69:906-910. 